\title{
UNIQUENESS CRITERIA FOR CONTINUOUS-TIME MARKOV CHAINS WITH GENERAL TRANSITION STRUCTURES
}

\author{
ANYUE CHEN, * University of Greenwich and the University of Hong Kong \\ PHIL POLLETT, ${ }^{* * * * *}$ \\ HANJUN ZHANG ****** AND \\ BEN CAIRNS, ${ }^{* * * * *}$ The University of Queensland
}

\begin{abstract}
We derive necessary and sufficient conditions for the existence of bounded or summable solutions to systems of linear equations associated with Markov chains. This substantially extends a famous result of G. E. H. Reuter, which provides a convenient means of checking various uniqueness criteria for birth-death processes. Our result allows chains with much more general transition structures to be accommodated. One application is to give a new proof of an important result of M. F. Chen concerning upwardly skip-free processes. We then use our generalization of Reuter's lemma to prove new results for downwardly skipfree chains, such as the Markov branching process and several of its many generalizations. This permits us to establish uniqueness criteria for several models, including the general birth, death, and catastrophe process, extended branching processes, and asymptotic birth-death processes, the latter being neither upwardly skip-free nor downwardly skipfree.
\end{abstract}

Keywords: Upwardly skip-free process; downwardly skip-free process; Markov branching process; birth-death process

2000 Mathematics Subject Classification: Primary 60J27

Secondary $60 \mathrm{~J} 35$

\section{Introduction}

We shall be concerned with continuous-time Markov chains that take values in a countable state space $S$. For convenience, we shall enumerate the states such that $S=\{0,1, \ldots\}$. We start with a stable, conservative $q$-matrix of transition rates over $S$, that is, a collection $\boldsymbol{Q}=\left(q_{i j}, i, j \in S\right)$ of real numbers that satisfies $0 \leq q_{i j}<\infty(j \neq i), q_{i}:=-q_{i i}<\infty$, and $\sum_{j \neq i} q_{i j} \leq q_{i}(i \in S)$. The matrix $\boldsymbol{Q}$ is said to be conservative if $\sum_{j \neq i} q_{i j}=q_{i}$ for all

Received 5 October 2004; revision received 6 June 2005.

* Current address: Department of Statistics and Actuarial Science, The University of Hong Kong, Pokfulam Road, Hong Kong. Email address: a.chen@hknstasc.hku.hk

** Postal address: Department of Mathematics, The University of Queensland, Qld 4072, Australia.

*** Email address: pkp@maths.uq.edu.au

**** Email address: hjz@maths.uq.edu.au

***** Current address: School of Biological Sciences, University of Bristol, Woodland Road, Clifton, Bristol BS8 1UG,

UK. Email address: Ben.Cairns@bristol.ac.uk 
$i \in S$. A set of real-valued functions $\boldsymbol{P}(\cdot)=\left(p_{i j}(\cdot), i, j \in S\right)$ defined on $[0, \infty)$ is called a standard transition function (or simply process) if

$$
\begin{gathered}
p_{i j}(t) \geq 0, \quad i, j \in S, t>0, \\
\sum_{j \in S} p_{i j}(t) \leq 1, \quad i \in S, t>0, \\
p_{i j}(s+t)=\sum_{k \in S} p_{i k}(s) p_{k j}(t), \quad i, j \in S, s, t>0,
\end{gathered}
$$

and $\lim _{t \downarrow 0} p_{i j}(t)=\delta_{i j}, i, j \in S$. The process $\boldsymbol{P}$ is then honest if equality holds in (1) for some (and, thus, all) $t>0$, and is called a $\boldsymbol{Q}$-transition function (or $\boldsymbol{Q}$-process) if $p_{i j}^{\prime}(0+)=q_{i j}$ for each $i, j \in S$.

When $\boldsymbol{Q}$ is conservative, every $\boldsymbol{Q}$-process $\boldsymbol{P}$ satisfies the backward differential equations,

$$
p_{i j}^{\prime}(t)=\sum_{k \in S} q_{i k} p_{k j}(t), \quad t>0,
$$

for all $i, j \in S$, but might not satisfy the forward differential equations,

$$
p_{i j}^{\prime}(t)=\sum_{k \in S} p_{i k}(t) q_{k j}, \quad t>0
$$

for all $i, j \in S$. Feller's recursion [12] provides for the existence of a minimal solution $\boldsymbol{F}(\cdot)=$ $\left(f_{i j}(\cdot), i, j \in S\right.$ ) to the backward equations that also satisfies the forward equations (and this is true whether or not $\boldsymbol{Q}$ is conservative). Indeed, $f_{i j}(t) \leq p_{i j}(t)$ for any $\boldsymbol{Q}$-transition function $\boldsymbol{P}$. It is the unique solution to the backward equations (among the nonnegative solutions) if and only if $\boldsymbol{Q}$ is regular, that is,

$$
(\lambda \boldsymbol{I}-\boldsymbol{Q}) \boldsymbol{u}(\lambda)=\mathbf{0}, \quad \boldsymbol{u}(\lambda) \in l_{\infty}^{+},
$$

has only the trivial solution for some (and, thus, all) $\lambda>0$ (here $l_{\infty}^{+}$denotes the set of nonnegative bounded sequences). When $\boldsymbol{Q}$ is conservative, this condition corresponds to $\boldsymbol{F}$ being honest, and it is necessary and sufficient for $\boldsymbol{F}$ to be the unique $\boldsymbol{Q}$-transition function [21]. When $\boldsymbol{Q}$ is not conservative, additional conditions are needed for $\boldsymbol{F}$ to be the unique $\boldsymbol{Q}$-transition function [15], [22], but condition (2) must certainly be checked first.

In applications involving continuous-time Markov chains, it is frequently necessary to determine whether or not $\boldsymbol{Q}$ is regular. For example, one might wish to study explosive behaviour of the process in question (the minimal process), or rule out such behaviour before proceeding further with any analysis. Alternatively, one might have an invariant probability measure $\boldsymbol{\pi}$ for $\boldsymbol{Q}$, and wish to determine whether $\boldsymbol{\pi}$ is invariant for the minimal transition function; here it is the regularity of related transition rates, rather than of $\boldsymbol{Q}$ itself, which must be established (see, for example, [18] and [20]).

When $\boldsymbol{F}$ is honest, it is also the unique solution to the forward equations, but when $\boldsymbol{F}$ is dishonest, uniqueness holds if and only if

$$
\boldsymbol{\eta}(\lambda)(\lambda \boldsymbol{I}-\boldsymbol{Q})=\mathbf{0}, \quad \eta(\lambda) \in l_{1}^{+},
$$

has only the trivial solution for some (and, thus, all) $\lambda>0$ (where $l_{1}^{+}$denotes the set of nonnegative summable sequences); again, see [21]. This latter condition arises in other contexts, 
for example in determining quasi-stationary distributions: if $\boldsymbol{Q}$ is regular and (3) has only the trivial solution, then all probability measures $\mu$-invariant for $\boldsymbol{Q}$ are also $\mu$-invariant for $\boldsymbol{F}$ [13], [14].

More delicate extensions of criteria (2) and (3) are possible to, for example, pure-jump Markov processes [10] and Markov chains with particular structures, such as upwardly skipfree chains [8], [9] and the multidimensional $Q$-processes considered in [23]; for a recent exposition, see Chapter 3 of [8].

A convenient means of dealing with (2) and (3) in the case of birth-death processes was provided in Reuter [21]. He proved the following simple result, which led to the various uniqueness criteria for birth-death processes.

Lemma 1. (Reuter [21].) Let $\left\{\sigma_{n}, n \geq 0\right\}$ be a sequence of real numbers satisfying $0 \leq \sigma_{0}<$ $\sigma_{1}$ and $\sigma_{n+1}-\sigma_{n}=f_{n} \sigma_{n}+h_{n}+g_{n}\left(\sigma_{n}-\sigma_{n-1}\right), n \geq 1$, where $\left\{f_{n}, n \geq 1\right\},\left\{h_{n}, n \geq 1\right\}$, and $\left\{g_{n}, n \geq 1\right\}$ are known nonnegative sequences. Then the sequence $\left\{\sigma_{n}, n \geq 1\right\}$ is bounded if and only if $\sum_{n=1}^{\infty}\left(F_{n}+H_{n}\right)<\infty$, where

$$
F_{n}=f_{n}+g_{n} f_{n-1}+\cdots+g_{n} g_{n-1} \cdots g_{2} f_{1}+g_{n} g_{n-1} \cdots g_{2} g_{1}
$$

and

$$
H_{n}=h_{n}+g_{n} h_{n-1}+\cdots+g_{n} g_{n-1} \cdots g_{2} h_{1} .
$$

For further details, see Section 3.2 of [1].

Reuter's lemma cannot be applied directly once the birth-death structure is lost, for example, in the case of upwardly skip-free chains, where there are additional downward transitions of any size; regularity conditions for upwardly skip-free chains were obtained in [23], [8], and [9]. Our aim here is to substantially extend Reuter's lemma in order to handle much more general transition structures. For example, our generalization provides a convenient means of establishing quasi-regularity (that there is exactly one honest $\boldsymbol{Q}$-process satisfying both the backward and the forward equations). We do this for downwardly skip-free processes, such as the Markov branching process and several of its many generalizations, as well as many processes that are neither upwardly skip-free nor downwardly skip-free.

The structure of the paper is as follows. The main result, our generalization of Reuter's lemma, is proved in Section 2. We illustrate this in Section 3 by providing an alternative proof of a special case of Theorem 1.1 of [9], which gives regularity conditions for upwardly skip-free processes. This is specialized to a population model: the general birth, death, and catastrophe process. In Section 4, downwardly skip-free processes are studied in some detail. This section includes a detailed analysis of extended branching processes. In Section 5, we introduce the notion of quasi-regularity and derive a means of identifying it. Finally, in Section 6, we study asymptotic birth-death processes, a class of Markov chains that are neither upwardly skip-free nor downwardly skip-free.

\section{A generalization of Reuter's lemma}

Our main result is the following.

Theorem 1. Let $\left\{\sigma_{n}, n \geq 0\right\}$ be a sequence of real numbers satisfying $0 \leq \sigma_{0}<\sigma_{1}$ and

$$
\sigma_{n+1}-\sigma_{n}=f_{n} \sigma_{n}+h_{n}+\sum_{m=1}^{n} g_{n m}\left(\sigma_{m}-\sigma_{m-1}\right), \quad n \geq 1,
$$


where $\left\{f_{n}, n \geq 1\right\},\left\{h_{n}, n \geq 1\right\}$, and $\left\{g_{n m}, n \geq 1,1 \leq m \leq n\right\}$ are all nonnegative. Then $\left\{\sigma_{n}\right\}$ is bounded if and only if

$$
\sum_{n=1}^{\infty} R_{n}<\infty
$$

where $\left\{R_{n}, n \geq 1\right\}$ is defined recursively by $R_{1}=r_{1}$ and

$$
R_{n}=r_{n}+\sum_{m=2}^{n} g_{n m} R_{m-1}, \quad n \geq 2,
$$

with

$$
r_{n}=f_{n}+h_{n}+g_{n 1}, \quad n \geq 1 .
$$

Proof. First, observe that $\sigma_{n}$ is increasing and strictly positive for $n \geq 1$. Next, define individual sequences $\left\{F_{n}, n \geq 1\right\},\left\{H_{n}, n \geq 1\right\}$, and $\left\{G_{n}, n \geq 1\right\}$ by

$$
\begin{array}{ll}
F_{1}=f_{1}, & F_{n}=f_{n}+\sum_{m=2}^{n} g_{n m} F_{m-1}, \quad n \geq 2, \\
H_{1}=h_{1}, & H_{n}=h_{n}+\sum_{m=2}^{n} g_{n m} H_{m-1}, \quad n \geq 2, \\
G_{1}=g_{11}, & G_{n}=g_{n 1}+\sum_{m=2}^{n} g_{n m} G_{m-1}, \quad n \geq 2,
\end{array}
$$

meaning that $R_{n}=F_{n}+G_{n}+H_{n}$. We will prove, by induction, that

$$
\left(F_{n}+G_{n}\right)\left(\sigma_{1}-\sigma_{0}\right)+H_{n} \leq \sigma_{n+1}-\sigma_{n} \leq\left(F_{n}+G_{n}\right) \sigma_{n}+H_{n}
$$

for all $n \geq 1$. Our aim then will be to deduce condition (7) by summing over $n$.

It is easily seen that (10) holds for $n=1$ since, because $\sigma_{0} \geq 0$,

$$
\sigma_{2}-\sigma_{1}=f_{1} \sigma_{1}+h_{1}+g_{11}\left(\sigma_{1}-\sigma_{0}\right) \leq f_{1} \sigma_{1}+h_{1}+g_{11} \sigma_{1}=\left(F_{1}+G_{1}\right) \sigma_{1}+H_{1}
$$

and

$$
\sigma_{2}-\sigma_{1} \geq f_{1}\left(\sigma_{1}-\sigma_{0}\right)+h_{1}+g_{11}\left(\sigma_{1}-\sigma_{0}\right)=\left(F_{1}+G_{1}\right)\left(\sigma_{1}-\sigma_{0}\right)+H_{1} .
$$

So, assume that

$$
\left(F_{k}+G_{k}\right)\left(\sigma_{1}-\sigma_{0}\right)+H_{k} \leq \sigma_{k+1}-\sigma_{k} \leq\left(F_{k}+G_{k}\right) \sigma_{k}+H_{k}
$$

holds for all $k \leq n-1$. Then,

$$
\begin{aligned}
\sigma_{n+1}-\sigma_{n} & =f_{n} \sigma_{n}+h_{n}+\sum_{m=2}^{n} g_{n m}\left(\sigma_{m}-\sigma_{m-1}\right)+g_{n 1}\left(\sigma_{1}-\sigma_{0}\right) \\
& \leq f_{n} \sigma_{n}+h_{n}+\sum_{m=2}^{n} g_{n m}\left(\left(F_{m-1}+G_{m-1}\right) \sigma_{m-1}+H_{m-1}\right)+g_{n 1}\left(\sigma_{1}-\sigma_{0}\right) \\
& \leq\left(f_{n}+\sum_{m=2}^{n} g_{n m} F_{m-1}\right) \sigma_{n}+h_{n}+\sum_{m=2}^{n} g_{n m} H_{m-1}+\left(g_{n 1}+\sum_{m=2}^{n} g_{n m} G_{m-1}\right) \sigma_{n} \\
& =\left(F_{n}+G_{n}\right) \sigma_{n}+H_{n}
\end{aligned}
$$


and, similarly,

$$
\begin{aligned}
\sigma_{n+1}-\sigma_{n} \geq & f_{n} \sigma_{n}+h_{n}+\sum_{m=2}^{n} g_{n m}\left(\left(F_{m-1}+G_{m-1}\right)\left(\sigma_{1}-\sigma_{0}\right)+H_{m-1}\right)+g_{n 1}\left(\sigma_{1}-\sigma_{0}\right) \\
\geq & h_{n}+\sum_{m=2}^{n} g_{n m} H_{m-1}+\left(f_{n}+\sum_{m=2}^{n} g_{n m} F_{m-1}\right)\left(\sigma_{1}-\sigma_{0}\right) \\
& +\left(g_{n 1}+\sum_{m=2}^{n} g_{n m} G_{m-1}\right)\left(\sigma_{1}-\sigma_{0}\right) \\
= & H_{n}+\left(F_{n}+G_{n}\right)\left(\sigma_{1}-\sigma_{0}\right) .
\end{aligned}
$$

Therefore, (11) holds for all $k \leq n$ and the induction is complete.

Next, we will show that $\left\{\sigma_{n}\right\}$ is bounded if and only if

$$
\sum_{n=1}^{\infty}\left(F_{n}+G_{n}+H_{n}\right)<\infty
$$

If $\left\{\sigma_{n}\right\}$ is bounded then (12) follows immediately, from the first inequality of (10) together with the fact that $0 \leq \sigma_{0}<\sigma_{1}$. Conversely, using the second inequality of (10), and remembering that $\left\{\sigma_{n}\right\}$ is increasing, we have

$$
\frac{\sigma_{n+1}}{\sigma_{n}}-1 \leq F_{n}+G_{n}+\frac{H_{n}}{\sigma_{n}} \leq F_{n}+G_{n}+\frac{H_{n}}{\sigma_{1}} .
$$

Hence, if (12) holds then

$$
\sum_{n=1}^{\infty}\left(\frac{\sigma_{n+1}}{\sigma_{n}}-1\right)<\infty
$$

However, this is equivalent to $\left\{\sigma_{n}\right\}$ being bounded, for if we set $x_{n}=\sigma_{n+1} / \sigma_{n}-1$ (which is nonnegative), then

$$
\frac{\sigma_{n+1}}{\sigma_{1}}=\prod_{m=1}^{n} \frac{\sigma_{m+1}}{\sigma_{m}}=\prod_{m=1}^{n}\left(1+x_{m}\right),
$$

and, so, $\left\{\sigma_{n}\right\}$ converges if and only if $\sum_{m=1}^{\infty} x_{m}<\infty$. This completes the proof.

In order to check condition (7), we must first evaluate $\left\{F_{n}\right\},\left\{H_{n}\right\}$, and $\left\{G_{n}\right\}$. The following simple result establishes a necessary condition for $\left\{\sigma_{n}\right\}$ to be bounded, in terms of the original sequences $\left\{f_{n}\right\},\left\{h_{n}\right\}$, and $\left\{g_{n m}\right\}$.

Corollary 1. If $\left\{\sigma_{n}\right\}$ in Theorem 1 is bounded then

$$
\sum_{n=1}^{\infty} r_{n}<\infty
$$

where $r_{n}=f_{n}+h_{n}+g_{n 1}$, and

$$
\sum_{n=m}^{\infty} g_{n m}<\infty \text { for all } m \geq 1
$$


Proof. Since $R_{n} \geq r_{n}$, (7) implies (13), which in turn implies that (14) holds for $m=1$. Suppose that (14) fails for $m=m_{0} \geq 2$. Then the sequence of partial sums $\left\{\sum_{n=m_{0}}^{k} g_{n m_{0}}\right\}$ is unbounded. Now, by (8) we have

$$
\sum_{n=2}^{k} R_{n} \geq \sum_{n=2}^{k} \sum_{m=2}^{n} g_{n m} R_{m-1}=\sum_{m=2}^{k} R_{m-1} \sum_{n=m}^{k} g_{n m} \geq R_{m_{0}-1} \sum_{n=m_{0}}^{k} g_{n m_{0}}
$$

for $k>m_{0}$, and, thus, $\left\{\sum_{n=2}^{k} R_{n}\right\}$ is also unbounded. This contradicts (7).

Remark 1. If we set $g_{n 1}=g_{n 2}=\cdots=g_{n, n-1}=0$ for all $n \geq 1$, then $G_{n} \equiv 0$ and $\left\{F_{n}\right\}$ and $\left\{H_{n}\right\}$ are now given by (4) and (5), respectively. Hence, Theorem 1 reduces to Reuter's result, Lemma 1.

Remark 2. In many practical situations, while it might not be possible to evaluate $\left\{R_{n}\right\}$ explicitly, it might still be possible to check (7) indirectly. For example, if $r_{n}^{\prime} \sim a r_{n}$ (as $n \rightarrow \infty)$, where $a>0$ and $\left\{r_{n}\right\}$ is given by (9), then (7) will hold if and only if $\sum_{n=1}^{\infty} R_{n}^{\prime}<\infty$, where

$$
R_{1}^{\prime}=r_{1}^{\prime} \quad \text { and } \quad R_{n}^{\prime}=r_{n}^{\prime}+\sum_{m=2}^{n} g_{n m} R_{m-1}^{\prime}
$$

\section{Upwardly skip-free chains}

Upwardly skip-free chains have been studied by several authors, most particularly, in the present context, Chen [8], [9] and Yan and Chen [23], but also Brockwell et al. [4], Brockwell [2], [3], Pakes [17], Pollett [19], J. K. Zhang [24], and Y. H. Zhang [25].

Definition 1. A conservative $q$-matrix $Q=\left(q_{i j}, i, j \in \mathbb{N}_{+}\right)$defined on the nonnegative integers $\mathbb{N}_{+}$is called upwardly skip-free if $q_{i, i+1}>0$ for all $i \geq 1$, and $q_{i j}=0$ for $i$ and $j$ such that $j>i+1$.

We will illustrate the utility of Theorem 1 by proving a special case of Theorem 1.1 of Chen [9] (Chen's result allows $q_{k, k+1}=0$ for finitely many $k$ ).

Theorem 2. (Chen [9].) Let $\boldsymbol{Q}=\left(q_{i j}, i, j \in \mathbb{N}_{+}\right)$be an upwardly skip-free q-matrix. Then $Q$ is regular if and only if

$$
\sum_{n=1}^{\infty} R_{n}=\infty
$$

where $R_{0}=1$ and, for $n \geq 1$,

$$
R_{n}=\frac{1}{q_{n, n+1}}\left(1+\sum_{m=1}^{n} \sum_{k=0}^{m-1} q_{n k} R_{m-1}\right)
$$

Proof. We will prove that (15) holds if and only if

$$
(\lambda \boldsymbol{I}-\boldsymbol{Q}) \boldsymbol{u}(\lambda)=\mathbf{0}, \quad \boldsymbol{u}(\lambda) \in l_{\infty}^{+},
$$

has only the trivial solution for some (and, thus, all) $\lambda>0$. When $\lambda=1$, (17) becomes $u_{i}=\sum_{k=0}^{i+1} q_{i k} u_{k}, i \geq 0$, and, after a little algebra, we find that

$$
q_{i, i+1}\left(u_{i+1}-u_{i}\right)=u_{i}+\sum_{m=1}^{i} \sum_{k=0}^{m-1} q_{i k}\left(u_{m}-u_{m-1}\right) .
$$


Thus, if we set $g_{n m}=\sum_{k=0}^{m-1} q_{n k} / q_{n, n+1}$ then $u_{1}=\left(1+1 / q_{01}\right) u_{0}$ and, for $n \geq 1$,

$$
u_{n+1}-u_{n}=\frac{1}{q_{n, n+1}} u_{n}+\sum_{m=1}^{n} g_{n m}\left(u_{m}-u_{m-1}\right) \text {. }
$$

It is easy to see that if $u_{0}=0$ then $u_{n} \equiv 0$. If $u_{0}>0$, we may identify $\left\{u_{n}\right\}$ with $\left\{\sigma_{n}\right\}$ in Theorem 1, setting $f_{n}=1 / q_{n, n+1}$ and $h_{n} \equiv 0$. On evaluating $R_{n}$ using (8) and (9), we obtain

$$
R_{1}=r_{1}=\frac{1}{q_{12}}\left(1+q_{10}\right), \quad r_{n}=\frac{1}{q_{n, n+1}}\left(1+q_{n 0}\right), \quad n \geq 2,
$$

and

$$
R_{n}=\frac{1}{q_{n, n+1}}\left(1+q_{n 0}\right)+\sum_{m=2}^{n} \frac{1}{q_{n, n+1}} \sum_{k=0}^{m-1} q_{n k} R_{m-1}, \quad n \geq 2,
$$

which are easily seen to correspond to (16). We conclude that $\left\{u_{n}\right\}$ is bounded (that is, (17) has a nontrivial solution) if and only if $\sum_{n=1}^{\infty} R_{n}<\infty$, and the proof is complete.

Remark 3. If $\boldsymbol{Q}$ is not regular, that is, $\sum_{n=1}^{\infty} R_{n}<\infty$, then $\boldsymbol{Q}$ is single-exit, because (17) has an essentially unique positive solution when $u_{0}>0$. Hence, since $\boldsymbol{Q}$ is conservative, there exists a unique honest $\boldsymbol{Q}$-process satisfying the backward equations (see, for example, Theorem 4.2.6(2) of [1]).

The following two corollaries provide conditions that are easier to check than (15) by way of (16).

Corollary 2. For the upwardly skip-free q-matrix $\boldsymbol{Q}$, let $\lambda_{n}=q_{n, n+1}, n \geq 0$, and $\mu_{n}=$ $\sum_{k=0}^{n-1} q_{n k}, n \geq 1$, and define

$$
R=\sum_{n=1}^{\infty}\left(\frac{1}{\lambda_{n}}+\frac{\mu_{n}}{\lambda_{n} \lambda_{n-1}}+\frac{\mu_{n} \mu_{n-1}}{\lambda_{n} \lambda_{n-1} \lambda_{n-2}}+\cdots+\frac{\mu_{n} \cdots \mu_{2}}{\lambda_{n} \cdots \lambda_{2} \lambda_{1}}\right) .
$$

Then each of the following conditions is sufficient for $\boldsymbol{Q}$ to be regular.

(i) $R=\infty$.

(ii) $\sum_{n=1}^{\infty} 1 / \lambda_{n}=\infty$.

(iii) There exists an $N$ such that $\mu_{n} \geq \lambda_{n}$ for all $n \geq N$.

Proof. We will prove that (15) holds under each of the stated conditions. From (16), we have $R_{n} \geq\left(1+\mu_{n} R_{n-1}\right) / \lambda_{n}$, which implies that

$$
R_{n} \geq \frac{1}{\lambda_{n}}+\frac{\mu_{n}}{\lambda_{n} \lambda_{n-1}}+\frac{\mu_{n} \mu_{n-1}}{\lambda_{n} \lambda_{n-1} \lambda_{n-2}}+\cdots+\frac{\mu_{n} \cdots \mu_{2}}{\lambda_{n} \cdots \lambda_{2} \lambda_{1}} .
$$

Condition (i) then follows from Theorem 3.2.2 of [1], if $\lambda_{n}$ and $\mu_{n}$ are identified as the rates of a birth-death process. Similarly, condition (ii) holds because $R_{n} \geq 1 / \lambda_{n}$ and condition (iii) because, for all $n \geq N, R_{n} \geq 1 / \lambda_{n}+R_{n-1} \geq R_{n-1}$ and, hence, $R_{n} \geq R_{N-1}$.

Corollary 3. (i) If there exists a nonnegative sequence $\left\{v_{i}, i \geq 1\right\}$ such that $\sum_{i=1}^{\infty} v_{i} \geq 1$ and

$$
\frac{1}{q_{n, n+1}} \sum_{k=0}^{m} q_{n k} \geq v_{n-m}, \quad n>m \geq 0,
$$

then $Q$ is regular. 
(ii) If there exists a nonnegative sequence $\left\{v_{i}, i \geq 1\right\}$ such that

$$
\frac{1}{q_{n, n+1}} \sum_{k=0}^{m} q_{n k}=v_{n-m}, \quad n>m \geq 0,
$$

then $Q$ is regular if and only if $\sum_{n=1}^{\infty} 1 / q_{n, n+1}=\infty$ or $\sum_{n=1}^{\infty} v_{n} \geq 1$.

Proof. To prove claim (i), set $g_{n}=1 / q_{n, n+1}$, meaning that (16) can be written

$$
R_{n}=g_{n}+\sum_{m=0}^{n-1} R_{m}\left(g_{n} \sum_{k=0}^{m} q_{n k}\right), \quad n \geq 1
$$

(remembering that $R_{0}=1$ ), and write $C=\sum_{n=0}^{\infty} R_{n}$. Now, (18) implies that

$$
R_{n} \geq g_{n}+\sum_{m=0}^{n-1} R_{m} v_{n-m}, \quad n \geq 1,
$$

and, so, in particular, $R_{n} \geq g_{n}$ and $R_{n} \geq v_{n}$. Thus, if $C<\infty$ then $G:=\sum_{n=1}^{\infty} g_{n}$ and $V:=\sum_{n=1}^{\infty} v_{n}$ are both finite and $C \geq 1+G+C V$, implying that $V<1$. Hence, by Theorem 2, having $V \geq 1$ implies that $\boldsymbol{Q}$ is regular.

Similarly, if (19) is satisfied, we now have

$$
R_{n}=g_{n}+\sum_{m=0}^{n-1} R_{m} v_{n-m}, \quad n \geq 1
$$

Thus, if $\boldsymbol{Q}$ is not regular, that is, $C<\infty$, then, as before, $G<\infty$ and $V<1$. Conversely, if $G<\infty$ and $V<1$ then, on summing (20) over $n$, we find that

$$
\sum_{n=1}^{k} R_{n}=\sum_{n=1}^{k} g_{n}+\sum_{m=0}^{k-1} R_{m} \sum_{n=1}^{k-m} v_{n} \leq G+V \sum_{m=0}^{k-1} R_{m}, \quad k \geq 1,
$$

which implies that $R_{k}+(1-V) \sum_{n=0}^{k-1} R_{n} \leq 1+G$ and, hence, that $C<\infty$.

We now give some examples to demonstrate the usefulness of these results.

Example 1. First we will consider a variant of the birth, death, and catastrophe process with 'binomial catastrophes' described in [4]. The $q$-matrix has elements

$$
q_{i j}= \begin{cases}w_{i}(1-p)^{i} & \text { if } j=i+1, i \geq 0 \\
-w_{i} & \text { if } j=i, i \geq 0 \\
w_{i}\left(\begin{array}{c}
i \\
i-j
\end{array}\right) p^{i-j}(1-p)^{j} & \text { if } 0 \leq j<i, i \geq 1 \\
0 & \text { otherwise }\end{cases}
$$

where $w_{0} \geq 0, w_{i}>0$ for all $i \geq 1$, and $0<p<1$. Thus, events occur at a rate $w_{i}$, which depends on the current state $i$, and the catastrophe size $(1,2, \ldots, i$, or -1 for a birth) is determined by the binomial distribution $\operatorname{bin}(i, p)$. If $w_{0}>0$ then the process is irreducible, 
while if $w_{0}=0$, there is a single absorbing state 0 , which is accessible from the irreducible class $\{1,2, \ldots\}$. We will show that $\boldsymbol{Q}$ is always regular.

In the notation of Corollary $2, \lambda_{n}=w_{n}(1-p)^{n}$ and

$$
\mu_{n}=\sum_{k=0}^{n-1} q_{n k}=w_{n} \sum_{k=0}^{n-1}\left(\begin{array}{c}
n \\
n-k
\end{array}\right) p^{n-k}(1-p)^{k}=w_{n}\left(1-(1-p)^{n}\right) .
$$

Thus, for a fixed $p$ in $(0,1)$, we have $1-(1-p)^{n} \geq(1-p)^{n}$ for $n$ sufficiently large. Hence, by condition (iii) of Corollary $2, \boldsymbol{Q}$ is regular.

Example 2. Our next example illustrates that, even when conditions (ii) and (iii) of Corollary 2 both fail to hold, the upwardly skip-free $q$-matrix may still be regular. Let $\boldsymbol{Q}$ be conservative with off-diagonal elements

$$
q_{i j}= \begin{cases}\frac{b}{2}(i+1)^{2} & \text { if } j=i+1 \\ d(i-j) & \text { if } 0 \leq j \leq i-1,\end{cases}
$$

where $0<b \leq d$. We now have $\sum_{n=1}^{\infty} 1 / q_{n, n+1}<\infty$. The birth and death rates of the corresponding birth-death $q$-matrix are respectively given by

$$
\lambda_{n}=\frac{b}{2}(n+1)^{2} \quad \text { and } \quad \mu_{n}=\frac{d}{2} n(n+1) .
$$

It is easy to see that when $d>b$ we may still apply condition (iii) of Corollary 2 to deduce that $Q$ is regular, because $d / b \geq 1+1 / n$ for all $n$ sufficiently large. When $d=b$, condition (ii) will always fail; however, when $d=b$, condition (i) of Corollary 2 implies that $\boldsymbol{Q}$ is regular, because

$$
R=\sum_{n=1}^{\infty}\left(\frac{2}{b(n+1)^{2}}+\frac{2 d}{b^{2}(n+1) n}+\cdots+\frac{2 d^{n-1}}{b^{n}(n+1) 2}\right)>\frac{1}{b} \sum_{n=2}^{\infty} \frac{1}{n}=\infty .
$$

Finally, let us illustrate Corollary 3.

Example 3. We will consider a special case of the general birth, death, and catastrophe process set out in [3]. Its $q$-matrix has elements

$$
q_{i j}= \begin{cases}w_{i} b & \text { if } j=i+1, i \geq 0, \\ -w_{i} & \text { if } j=i, i \geq 0, \\ w_{i} a_{i-j} & \text { if } j=1,2, \ldots, i-1, i \geq 2, \\ w_{i} \sum_{k=i}^{\infty} a_{k} & \text { if } j=0, i \geq 1, \\ 0 & \text { otherwise, }\end{cases}
$$

where $w_{0} \geq 0, w_{i}>0$ for all $i \geq 1, b>0, a_{i}>0$ for least one $i \geq 1$, and $b+\sum_{i=1}^{\infty} a_{i}=1$. (If $a_{1}>0$ and $a_{i}=0$ for all $i \geq 2$, we recover the simple birth-death process with birth rates $w_{i} b$ and death rates $w_{i}(1-b)$.) If $w_{0}>0$ then the process is irreducible, while if $w_{0}=0$, there is a single absorbing state 0 , which is accessible from the irreducible class $\{1,2, \ldots\}$. 
Let $d$ be the probability-generating function defined by $d(s)=b+\sum_{i=1}^{\infty} a_{i} s^{i+1},|s|<1$, and let $B(s)=d(s)-s$. For the present model, Lemma 3.1 of [3] establishes that state 0 is reached with probability 1 if and only if the drift $D$, given by

$$
D=-B^{\prime}(1-)=b-\sum_{i=1}^{\infty} i a_{i}=1-\sum_{i=1}^{\infty}(i+1) a_{i},
$$

is less than or equal to 0 . Thus, the process cannot be explosive when $D \leq 0$. However, it can be explosive when $D>0$ : the simple birth-death process referred to immediately above is explosive if and only if $b>\frac{1}{2}$ (that is, $D>0$ ) and $\sum_{i=1}^{\infty} 1 / w_{i}<\infty$. It is clear from Corollary 2 that both (i) $\sum_{i=1}^{\infty} 1 / w_{i}=\infty$ and (ii) $b \leq \frac{1}{2}$ are sufficient conditions for regularity. However, we can do much better.

Theorem 3. The upwardly skip-free q-matrix $\boldsymbol{Q}$ given by (21) is regular if and only if

$$
\sum_{i=1}^{\infty} 1 / w_{i}=\infty \quad \text { or } \quad \sum_{i=1}^{\infty} i a_{i} \geq b .
$$

Proof. Set $v_{i}=(1 / b) \sum_{k=i}^{\infty} a_{k}, i \geq 1$. Then

$$
\frac{1}{q_{n, n+1}} \sum_{k=0}^{m} q_{n k}=v_{n-m}, \quad n>m \geq 0,
$$

and $\sum_{i=1}^{\infty} v_{i}=(1 / b) \sum_{i=1}^{\infty} i a_{i}$. Therefore, by claim (ii) of Corollary 3, $\boldsymbol{Q}$ is regular if and only if $\sum_{i=1}^{\infty} 1 / w_{i}=\infty$ or $\sum_{i=1}^{\infty} i a_{i} \geq b$.

Remark 4. In the case that $w_{0}=0$, there is a single absorbing state 0 . However, the result holds with a straightforward modification when there are $K$ absorbing states $\left(w_{0}=\right.$ $\left.w_{1}=\cdots=w_{K-1}=0\right)$ : the condition $\sum_{i=1}^{\infty} 1 / w_{i}=\infty$ is replaced by $\sum_{i=K}^{\infty} 1 / w_{i}=\infty$.

\section{Downwardly skip-free chains}

In this section, we will use Theorem 1 to obtain uniqueness criteria for downwardly skipfree chains. We will give particular attention to an important subclass of chains, the so-called extended branching processes.

Definition 2. A conservative $q$-matrix $\boldsymbol{Q}=\left(q_{i j}, i, j \in \mathbb{N}_{+}\right)$is called downwardly skip-free if $q_{i, i-1}>0$ for all $i \geq 1$ and $q_{i j}=0$ for $j<i-1$ and $i \geq 2$.

Theorem 4. For a downwardly skip-free q-matrix $\boldsymbol{Q}=\left(q_{i j}, i, j \in \mathbb{N}_{+}\right)$, the equation

$$
\boldsymbol{\eta}(\lambda)(\lambda \boldsymbol{I}-\boldsymbol{Q})=\mathbf{0}, \quad \boldsymbol{\eta}(\lambda) \in l_{1}^{+},
$$

has a nontrivial solution for some (and, thus, all) $\lambda>0$ if and only if $\sum_{n=1}^{\infty} R_{n}<\infty$, where $R_{0}=R_{-1}=1$ and, for all $n \geq 1$,

$$
R_{n}=\frac{1}{q_{n+1, n}}\left(1+\sum_{m=0}^{n} \sum_{k=n+1}^{\infty} q_{m k} R_{m-1}\right) .
$$


Proof. It is well known that the dimension of the solution space of (22) is the same for all $\lambda>0$ and, thus, can be denoted $\mathfrak{N}^{+}(\boldsymbol{Q})$. It is also clear that, since $\boldsymbol{Q}$ is downwardly skip-free, there are only two possibilities: either $\mathfrak{N}^{+}(\boldsymbol{Q})=0$ or $\mathfrak{N}^{+}(\boldsymbol{Q})=1$. So, fix $\lambda=1$ and let $\boldsymbol{\eta}(1)=\left(\eta_{0}, \eta_{1}, \eta_{2}, \ldots\right)$ be the (essentially unique) solution to $\boldsymbol{\eta}(1)(\boldsymbol{I}-\boldsymbol{Q})=\mathbf{0}$. Without loss of generality, set $\eta_{0} \equiv 1$. Then $\eta_{k}=\sum_{m=0}^{k+1} \eta_{m} q_{m k}, k \geq 0$, and, thus,

$$
\sum_{k=0}^{n} \eta_{k}=\sum_{k=0}^{n} \sum_{m=0}^{k+1} \eta_{m} q_{m k}
$$

On rearranging this, we obtain

$$
\eta_{n+1} q_{n+1, n}=\sum_{k=0}^{n} \eta_{k}+\eta_{0} \sum_{k=n+1}^{\infty} q_{0 k}+\sum_{m=1}^{n} \eta_{m} \sum_{k=n+1}^{\infty} q_{m k} .
$$

Thus, by setting $\sigma_{0}=\eta_{0} \equiv 1$ and $\sigma_{n}=\sum_{k=0}^{n} \eta_{k}, n \geq 1$, we see that $\sigma_{1}>\sigma_{0}=1$ and, for $n \geq 1$,

$$
\sigma_{n+1}-\sigma_{n}=\frac{1}{q_{n+1, n}} \sigma_{n}+\frac{\sigma_{0}}{q_{n+1, n}} \sum_{k=n+1}^{\infty} q_{0 k}+\frac{1}{q_{n+1, n}} \sum_{m=1}^{n} \sum_{k=n+1}^{\infty} q_{m k}\left(\sigma_{m}-\sigma_{m-1}\right),
$$

which is of the form (6) required in Theorem 1. After a little algebra, we conclude that $\left\{\sigma_{n}\right\}$ is bounded (that is, $\eta(1) \in l_{1}^{+}$) if and only if $\sum_{n=1}^{\infty} R_{n}<\infty$, and the proof is complete.

Remark 5. The significance of (22) was mentioned briefly in the introduction. If the minimal $\boldsymbol{Q}$-transition function $\boldsymbol{F}$ is honest, then $\boldsymbol{F}$ is the unique solution to the forward equations (and $\boldsymbol{Q}$ is necessarily conservative). However, when $\boldsymbol{F}$ is dishonest, it is the unique solution to the forward equations if and only if (22) has only the trivial solution. This is true whether or not $Q$ is conservative.

Corollary 4. For the downwardly skip-free q-matrix $\boldsymbol{Q}$, let $\mu_{n}=q_{n, n-1}, n \geq 1$, and $\lambda_{n}=$ $\sum_{k=n+1}^{\infty} q_{n k}, n \geq 0$, and define

$$
S=\sum_{n=1}^{\infty} \frac{1}{\mu_{n+1}}\left(1+\frac{\lambda_{n}}{\mu_{n}}+\frac{\lambda_{n} \lambda_{n-1}}{\mu_{n} \mu_{n-1}}+\cdots+\frac{\lambda_{n} \cdots \lambda_{2} \lambda_{1}}{\mu_{n} \cdots \mu_{2} \mu_{1}}\right)
$$

(not to be confused with the state space of the Markov chains). If $S=\infty$ then (22) has only the trivial solution. In particular, if $\sum_{n=1}^{\infty} 1 / \mu_{n+1}=\infty$ then (22) has only the trivial solution.

Proof. The proof is similar to that of Corollary 2, this time applying Theorem 3.2.3 of [1] to the birth-death process with birth and death rates $\lambda_{n}$ and $\mu_{n}$.

Example 4. We now consider an important subclass of downwardly skip-free processes: the 'extended' branching processes discussed in [11]. For simplicity, we will only consider the absorbing case, where the $q$-matrix $\boldsymbol{Q}=\left(q_{i j}\right)$ satisfies $q_{0 j} \equiv 0$. Here, $\boldsymbol{Q}$ is downwardly skip-free and

$$
q_{i j}= \begin{cases}w_{i} b_{j-i+1} & \text { if } j \geq i-1, i \geq 1 \\ 0 & \text { otherwise }\end{cases}
$$

where the sequence $\left\{b_{j}\right\}$ satisfies $b_{0}>0, b_{j} \geq 0$ for $j \geq 2$, and $-b_{1}=\sum_{j \neq 1} b_{j}>0$, and the sequence $\left\{w_{j}\right\}$ satisfies $w_{0}=0, w_{i}>0$ for $i \geq 1$, and (without loss of generality) $w_{1}=1$. Applying Theorem 4 immediately yields the following result. 
Corollary 5. For the extended branching $q$-matrix $\boldsymbol{Q}$ given by (23), the following statements hold.

(i) Either $\mathfrak{N}^{+}(\boldsymbol{Q})=0$ or $\mathfrak{N}^{+}(\boldsymbol{Q})=1$.

(ii) $\mathfrak{N}^{+}(\boldsymbol{Q})=0$ if and only if $\sum_{n=1}^{\infty} R_{n}=\infty$, where $R_{0}=1$ and, for $n \geq 1$,

$$
R_{n}=\frac{1}{b_{0} w_{n+1}}\left(1+\sum_{m=1}^{n} w_{m} \tau_{n+2-m} R_{m-1}\right)
$$

with $\tau_{k}=\sum_{j=k}^{\infty} b_{j}, k \geq 2$. In particular, if $\sum_{n=1}^{\infty} 1 / w_{n}=\infty$ then $\mathfrak{N}^{+}(\boldsymbol{Q})=0$.

Remark 6. The latter sufficient condition, $\sum_{n=1}^{\infty} 1 / w_{n}=\infty$, is not very sharp, but it does accommodate the ordinary Markov branching process, obtained on setting $w_{n}=n$ for all $n \geq 1$. If Harris's condition (Theorem 3.3.3(2) of [1]) fails, then there are infinitely many $\boldsymbol{Q}$-processes (including infinitely many honest ones). Corollary 5 establishes that there is only one, namely the minimal $\boldsymbol{Q}$-process, that satisfies the forward equations, and, hence, only one that has the 'branching property' (Theorem 3.3.1(2) of [1]). When $\sum_{n=1}^{\infty} 1 / w_{n}<\infty$, the situation is considerably more delicate, as our next theorem demonstrates.

Define the generating function of the sequence $\left\{b_{j}\right\}$ by $B(s)=\sum_{j=0}^{\infty} b_{j} s^{j}$. Recall that $B$ is a convex function on $(0,1]$ and, thus, has a smallest zero $q \in(0,1]$ that satisfies $q=1$ if $B^{\prime}(1) \leq 0$ and $q<1$ if $B^{\prime}(1)>0$. Furthermore, $B(s)>0$ for all $s \in[0, q)$. Note that $B^{\prime}(1)>0$ includes the important case $B^{\prime}(1)=\infty$. Henceforth, $q$ will always denote the smallest zero of $B$ on $(0,1]$.

Theorem 5. For the extended branching q-matrix $\boldsymbol{Q}$ with $\sum_{n=1}^{\infty} 1 / w_{n}<\infty$, let $\bar{w}$ and $\underline{w}$ be, respectively, the limit supremum and the limit infimum of $\sqrt[n]{w_{n+1}}$ as $n \rightarrow \infty$. The following statements then hold.

(i) Suppose that $B^{\prime}(1) \leq 0$. If $\bar{w}<1$ then $\mathfrak{N}^{+}(\boldsymbol{Q})=0$, while if $\underline{w}>1$ then $\mathfrak{N}^{+}(\boldsymbol{Q})=1$. In particular, if $\lim _{n \rightarrow \infty} \sqrt[n]{w_{n+1}}=w$ exists then $\mathfrak{N}^{+}(\boldsymbol{Q})$ equals 1 if $w>1$ and 0 if $w<1$.

(ii) Suppose that $B^{\prime}(1)>0$ (including $B^{\prime}(1)=\infty$ ). If $\bar{w}<1 / q$ then $\mathfrak{N}^{+}(\boldsymbol{Q})=0$, while if $\underline{w}>1 / q$ then $\mathfrak{N}^{+}(\boldsymbol{Q})=1$. In particular, if $\lim _{n \rightarrow \infty} \sqrt[n]{w_{n+1}}=w$ exists then $\mathfrak{N}^{+}(\boldsymbol{Q})$ equals 1 if $w>1 / q$ and 0 if $w<1 / q$.

Proof. Let $\left\{R_{n}\right\}$ be as in Corollary 5 and let $T_{n}=w_{n+1} R_{n}, n \geq 1$, and $T_{0}=w_{1} R_{0}=$ $R_{0}=1$. Define the generating function of $\left\{T_{n}\right\}$ by $T(s)=\sum_{n=0}^{\infty} T_{n} s^{n}$. We will establish that $T(s)$ has radius of convergence $q$, that is, $\lim _{\sup } \rightarrow \infty \sqrt[n]{T_{n}}=1 / q$, by first proving that

$$
\limsup _{n \rightarrow \infty} \sqrt[n]{T_{n}} \geq \frac{1}{q}
$$

and

$$
\limsup _{n \rightarrow \infty} \sqrt[n]{T_{n}} \leq \frac{1}{q}
$$

If (25) is not true then the radius of convergence of $T(s)$ is strictly greater than $q$. Hence, there exists an $\varepsilon>0$ such that $T(s)<\infty$ for all $s \in[0, q+\varepsilon)$, meaning that, in particular, $T(q)<\infty$. Now, it is readily established that $T(s) B(s)=b_{0}(1-s)+s$ for all $s \in[0, q+\varepsilon)$. However, $T(q)<\infty$ and $B(q)=0$, so by setting $s=q$ we find that $b_{0}(1-q)+q=0$. This is a contradiction, and, hence, (25) holds. 
Similarly, if (26) is not true, then the radius of convergence of $T(s)$ is strictly less than $q$; hence, there is an $r \in(0, q)$ with $T(r)=\infty$. However, this is impossible, for, as we will now prove, $T(s)<\infty$ for all $s \in[0, q)$. We need only show that

$$
\sup _{k \geq 1} \sum_{n=1}^{k} T_{n} s^{n}<\infty
$$

for all $s \in[0, q)$. Using (24), we learn that, for any $s \in[0, q)$ and any $k \geq 1$,

$$
\begin{aligned}
\sum_{n=1}^{k} T_{n} s^{n} & =\frac{1}{b_{0}} \sum_{n=1}^{k} s^{n}+\frac{1}{b_{0}} \sum_{n=1}^{k} \sum_{m=1}^{n} T_{m-1} \tau_{n+2-m} s^{n} \\
& =\frac{1}{b_{0}} \sum_{n=1}^{k} s^{n}+\frac{1}{b_{0}} \sum_{m=1}^{k} T_{m-1} s^{m-2} \sum_{n=2}^{k-n+2} \tau_{n} s^{n} \\
& \leq \frac{s}{b_{0}(1-s)}+\frac{1}{b_{0}} \sum_{m=1}^{k} T_{m-1} s^{m-2} \sum_{n=2}^{\infty} \tau_{n} s^{n}
\end{aligned}
$$

However, it is clear that

$$
\sum_{n=2}^{\infty} \tau_{n} s^{n}=\sum_{n=2}^{\infty} \sum_{k=n}^{\infty} b_{k} s^{n}=b_{0} s-\left(\frac{s}{1-s}\right) B(s)
$$

and, hence, that

$$
B(s) \sum_{n=0}^{k-1} T_{n} s^{n} \leq b_{0}(1-s)+s .
$$

Since $s \in[0, q)$, we have $B(s)>0$ and, therefore,

$$
\sum_{n=0}^{k-1} T_{n} s^{n} \leq \frac{b_{0}(1-s)+s}{B(s)} .
$$

The right-hand side of this inequality is certainly finite and does not depend on $k$. Thus, (27) and, hence, (26) hold.

We have proved that $\limsup _{n \rightarrow \infty} \sqrt[n]{T_{n}}=1 / q$. However, $T_{n}=w_{n+1} R_{n}$ and, since both $\left\{w_{n}\right\}$ and $\left\{R_{n}\right\}$ are nonnegative, we have

$$
\limsup _{n \rightarrow \infty} \sqrt[n]{R_{n}} \liminf _{n \rightarrow \infty} \sqrt[n]{w_{n+1}} \leq \limsup _{n \rightarrow \infty} \sqrt[n]{T_{n}} \leq \limsup _{n \rightarrow \infty} \sqrt[n]{R_{n}} \limsup _{n \rightarrow \infty} \sqrt[n]{w_{n+1}}
$$

and, hence,

$$
\underline{w} \limsup _{n \rightarrow \infty} \sqrt[n]{R_{n}} \leq \frac{1}{q} \leq \bar{w} \limsup _{n \rightarrow \infty} \sqrt[n]{R_{n}}
$$

Now, if $\bar{w}<1 / q$ then the right-hand side of (28) implies that $\lim _{\sup _{n \rightarrow \infty}} \sqrt[n]{R_{n}}>1$. Thus, the radius of convergence of $\sum_{n=1}^{\infty} R_{n} s^{n}$ is strictly less than 1 and, therefore, $\sum_{n=1}^{\infty} R_{n}=\infty$. If $\underline{w}>1 / q$ then the left-hand side of (28) gives $\lim _{\sup _{n \rightarrow \infty}} \sqrt[n]{R_{n}}<1$, implying that the radius of convergence is strictly greater than 1 and, hence, that $\sum_{n=1}^{\infty} R_{n}<\infty$. The result follows, remembering that, for statement (i), $q=1$ when $B^{\prime}(1) \leq 0$. 
As a direct consequence of Theorem 5, we obtain the following result, which settles the question of uniqueness for a large class of extended branching process. Guided by the fact that, for an ordinary Markov branching process, the 'branching property' holds only if its transition function obeys the forward equations, we will say that a continuous-time Markov chain taking values in $\mathbb{N}_{+}$is an extended branching process if its $q$-matrix is of the form (23) and its transition function satisfies the forward equations.

Theorem 6. For the q-matrix $\boldsymbol{Q}$ defined by (23), the following statements hold.

(i) If $B^{\prime}(1) \leq 0$ then there is only one extended branching process, namely the minimal $Q$-process. It is honest.

(ii) If $B^{\prime}(1)>0$ and $q \bar{w}<1$, where $\bar{w}=\limsup _{n \rightarrow \infty} \sqrt[n]{w_{n+1}}$, then there is only one extended branching process, namely the minimal $Q$-process. It is dishonest.

(iii) If $B^{\prime}(1)>0$ and $q \underline{w}>1$, where $\underline{w}=\liminf _{n \rightarrow \infty} \sqrt[n]{w_{n+1}}$, then there are infinitely many extended branching processes, of which one is the minimal $Q$-process. Exactly one of these is honest, but it is not the minimal $Q$-process.

(iv) In particular, if $B^{\prime}(1)>0$ and $\lim _{n \rightarrow \infty} \sqrt[n]{w_{n+1}}=w$ exists, then if $q w<1$, there is only one extended branching process, which is the (dishonest) minimal $Q$-process, while if $q w>1$, there are infinitely many extended branching processes, one of which is the minimal $\boldsymbol{Q}$-process; exactly one is honest, but it is not the minimal $\boldsymbol{Q}$-process.

Proof. The proof of statement (i) can be found in [11]. The proofs of statement (ii) and the first part of statement (iii) follow from Corollary 5. Statement (iii) follows directly from Theorem 14.2.8 of [16]. Statement (iv) combines statements (ii) and (iii).

In many instances, we actually have $w:=\lim _{n \rightarrow \infty} \sqrt[n]{w_{n+1}}=1$. For example, for the so-called generalized Markov branching process discussed in [5], we have $w_{n}=n^{\theta}$, where $\theta>0$ and, hence, $w=1$. Thus, statements (i) and (ii) of Theorem 6 allow us to deduce that there is always a single generalized Markov branching process. It is the minimal $\boldsymbol{Q}$-process, whether or not $\boldsymbol{Q}$ is regular. On the other hand, it is easy to construct examples in which statement (iii) applies. For example, if we set $w_{n}=(1 / q+\epsilon)^{n}$, where $\epsilon>0$, then, recalling that $0<q<1$ because $B^{\prime}(1)>0$, we have $w=1 / q+\epsilon>1 / q$. The resulting unique, honest nonminimal extended branching process has many interesting properties, but we will not pursue this here.

We conclude this section with the following result, the proof of which is very similar to that of Theorem 4.

Theorem 7. If $\boldsymbol{Q}$ is a downwardly skip-free $q$-matrix, then it has a unique invariant measure, that is, $\boldsymbol{\pi} \boldsymbol{Q}=\mathbf{0}$ has an essentially unique positive solution. This satisfies $\sum_{i} \pi_{i}<\infty$ (and, hence, $\boldsymbol{Q}$ admits a unique invariant probability measure) if and only if

$$
\sum_{n=1}^{\infty} R_{n}<\infty
$$

where $R_{0}=R_{-1}=1$ and, for $n \geq 1$,

$$
R_{n}=\frac{1}{q_{n+1, n}} \sum_{m=0}^{n} \sum_{k=n+1}^{\infty} q_{m k} R_{m-1} .
$$




\section{Quasi-regularity}

In many situations, it is necessary to assume that the transition function in question satisfies both the backward and the forward equations. For example, Karlin and McGregor's integral representation of the transition function of a birth-death process is valid only when it satisfies both sets of equations (see, for example, Section 8.2 of [1]). Also, if $\boldsymbol{Q}$ is conservative (or, more generally, if the set of nonconservative states is finite), then the minimal transition function is the unique $\boldsymbol{Q}$-process if and only if it uniquely satisfies both sets of equations (Corollary 3.13 of [8]). However, if the $q$-matrix is not regular, then there may exist honest transition functions satisfying both. We are interested in determining when there is exactly one.

Definition 3. A conservative $q$-matrix $\boldsymbol{Q}$ is called quasi-regular if there exists exactly one honest $\boldsymbol{Q}$-process satisfying both the backward and the forward equations.

If $\boldsymbol{Q}$ is regular then it is quasi-regular, but the converse is not always true. It is therefore of interest to find conditions under which a nonregular $q$-matrix is quasi-regular. Such conditions were given in Chapter 14 of [16]. However, in many cases, and in particular for the model discussed in the next section, it is more convenient to identify quasi-regularity by studying certain restrictions of the $q$-matrix (such restrictions will usually be nonconservative).

Lemma 2. Suppose that $\boldsymbol{Q}$ is a conservative $q$-matrix over a countable state space $E$. Let $b \in E$ and let $\boldsymbol{Q}^{*}$ denote the restriction of $\boldsymbol{Q}$ to $E \backslash\{b\}$. Then the following statements hold.

(i) $Q$ is regular if and only if

$$
\left(\lambda \boldsymbol{I}-Q^{*}\right) \boldsymbol{u}(\lambda)=0, \quad \boldsymbol{u}(\lambda) \in l_{\infty}^{+}
$$

has only the trivial solution for some (and, thus, all) $\lambda>0$.

(ii) If $\boldsymbol{Q}$ is not regular then it is quasi-regular if and only if $\mathfrak{N}^{+}\left(\boldsymbol{Q}^{*}\right)=1$, that is,

$$
\eta(\lambda)\left(\lambda \boldsymbol{I}-Q^{*}\right)=0, \quad \eta(\lambda) \in l_{1}^{+},
$$

has one and only one linearly independent solution for some (and, thus, all) $\lambda>0$.

Lemma 2 can be proved using the resolvent decomposition theorem, refined in [6] and [7], together with Theorem 14.2.8 of [16]. For brevity, we shall omit the details.

The role of Theorem 1 in identifying quasi-regularity will now be clear. Certainly, if $Q^{*}$ is either upwardly or downwardly skip-free then we can, in principle, follow the programme laid out in the previous sections. However, note that $\boldsymbol{Q}$ itself need not be skip-free. An interesting class of Markov chain, which are neither upwardly skip-free nor downwardly skip-free, but which can be treated using Lemma 2, are the 'asymptotic birth-death processes'. These will be studied in detail in the next section.

\section{Asymptotic birth-death processes}

Definition 4. A conservative $q$-matrix $Q=\left(q_{i j}, i, j \in \mathbb{N}_{+}\right)$is called an asymptotic birthdeath $q$-matrix if there is a finite subset $G$ of $\mathbb{N}_{+}$for which the restriction of $\boldsymbol{Q}$ to $\mathbb{N}_{+} \backslash G$, denoted $\boldsymbol{Q}^{*}$, is a birth-death $q$-matrix. Each corresponding $\boldsymbol{Q}$-process is called an asymptotic birth-death process. 
For simplicity, we shall only consider the case $G=\{0\}$, but our conclusions hold, with obvious modifications, when $G$ is any finite subset. We may therefore assume that $Q^{*}=$ $\left(q_{i j}, i, j \geq 1\right)$ takes the form

$$
q_{i j}= \begin{cases}a_{i} & \text { if } j=i-1, i \geq 2 \\ b_{i} & \text { if } j=i+1, i \geq 1, \\ -\left(a_{i}+b_{i}+d_{i}\right) & \text { if } j=i \geq 1 \\ 0 & \text { otherwise }\end{cases}
$$

where $a_{i}>0, b_{i}>0$, and $d_{i} \geq 0, i \geq 1$. Since $Q$ is assumed to be conservative, we may write the entries $\left(q_{i 0}, i \geq 1\right)$ in terms of $Q^{*}$.

The next two theorems provide a means of checking regularity and quasi-regularity for asymptotic birth-death processes.

Theorem 8. For the nonconservative birth-death q-matrix $\boldsymbol{Q}^{*}$ given in (31), (29) has only the trivial solution if and only if

$$
R:=\sum_{n=1}^{\infty}\left(\frac{1+d_{n}}{b_{n}}+\frac{a_{n}\left(1+d_{n-1}\right)}{b_{n} b_{n-1}}+\frac{a_{n} a_{n-1}\left(1+d_{n-2}\right)}{b_{n} b_{n-1} b_{n-2}}+\cdots+\frac{a_{n} a_{n-1} \cdots a_{2}\left(1+d_{1}\right)}{b_{n} b_{n-1} \cdots b_{1}}\right)=\infty
$$

Proof. On substituting (31) into (29) we find that $u_{2}(\lambda)=\left(\lambda+a_{1}+b_{1}+d_{1}\right) u_{1}(\lambda) / b_{1}$ and

$$
u_{n+1}(\lambda)=\left(\frac{\lambda+a_{n}+b_{n}+d_{n}}{b_{n}}\right) u_{n}(\lambda)-\frac{a_{n}}{b_{n}} u_{n-1}(\lambda), \quad n \geq 2 .
$$

Hence, $u_{2}(\lambda)-u_{1}(\lambda)=\left(\lambda+a_{1}+d_{1}\right) u_{1}(\lambda) / b_{1}$ and

$$
u_{n+1}(\lambda)-u_{n}(\lambda)=\left(\frac{\lambda+d_{n}}{b_{n}}\right) u_{n}(\lambda)+\frac{a_{n}}{b_{n}}\left(u_{n}(\lambda)-u_{n-1}(\lambda)\right), \quad n \geq 2,
$$

which is of the form (6) required by Theorem 1 . If $u_{1}(\lambda)>0$ then $u_{i}(\lambda)>0$ for all $i$ and, after evaluating $\left\{R_{n}\right\}$ using (8), we find that $R$ (above) is equal to $\sum_{n=1}^{\infty} R_{n}$. Thus, $\left\{u_{i}(\lambda)\right\}$ is bounded if and only if $R<\infty$, and the result follows.

Observe that Reuter's result, Lemma 1, is enough to prove Theorem 8 . However, we certainly need Theorem 1 to obtain conditions under which (30) has exactly one solution.

Theorem 9. For the nonconservative birth-death q-matrix $\boldsymbol{Q}^{*}$ given in (31), (30) has only the trivial solution if and only if $S:=\sum_{n=1}^{\infty} S_{n}=\infty$, where $S_{0}=0, S_{1}=1 / a_{2}$, and

$$
S_{n+1}=\left(\frac{a_{n+1}+b_{n+1}+d_{n+1}}{a_{n+2}}\right) S_{n}-\frac{b_{n}}{a_{n+2}} S_{n-1}, \quad n \geq 1 .
$$

Furthermore, $\mathfrak{N}^{+}\left(\boldsymbol{Q}^{*}\right)=1$ if and only if $\sum_{n=1}^{\infty} S_{n}<\infty$.

Proof. Let $\boldsymbol{\eta}=\left(\eta_{i}, i \geq 1\right)$ be any nonnegative solution of $\boldsymbol{\eta}(\lambda)\left(\lambda \boldsymbol{I}-\boldsymbol{Q}^{*}\right)=\mathbf{0}$ corresponding to $\lambda=1$. This satisfies $\left(1+a_{1}+b_{1}+d_{1}\right) \eta_{1}=a_{2} \eta_{2}$ and

$$
\left(1+a_{n}+b_{n}+d_{n}\right) \eta_{n}=a_{n+1} \eta_{n+1}+b_{n-1} \eta_{n-1}, \quad n \geq 2 .
$$


Since $\eta_{1}=0$ implies that $\eta_{n}=0$ for all $n \geq 1$, we shall assume that $\eta_{1}>0$. Then, setting $\sigma_{0}=0$ and $\sigma_{n}=\sum_{m=1}^{n} \eta_{m}$ for $n \geq 1$, we obtain

$$
\begin{aligned}
\sigma_{n+1}-\sigma_{n}=\frac{1}{a_{n+1}} \sigma_{n}+\frac{1}{a_{n+1}}( & \left(a_{1}+d_{1}\right)\left(\sigma_{1}-\sigma_{0}\right) \\
& \left.+\sum_{m=2}^{n-1} d_{m}\left(\sigma_{m}-\sigma_{m-1}\right)+\left(d_{n}+b_{n}\right)\left(\sigma_{n}-\sigma_{n-1}\right)\right)
\end{aligned}
$$

for all $n \geq 1$, noting that $\sigma_{1}>\sigma_{0}=0$. Hence, by Theorem $1,\left\{\sigma_{n}\right\}$ is bounded (that is, $\left.\sum_{n=1}^{\infty} \eta_{n}<\infty\right)$ if and only if $\sum_{n=1}^{\infty} R_{n}<\infty$, where $R_{1}=r_{1}$ and

$$
R_{n}=r_{n}+\sum_{m=2}^{n-1} \frac{d_{m}}{a_{n+1}} R_{m-1}+\frac{d_{n}+b_{n}}{a_{n+1}} R_{n-1}, \quad n \geq 2,
$$

with $r_{n}=\left(1+a_{1}+d_{1}\right) / a_{n+1}, n \geq 1$. However, in view of Remark $2, \sum_{n=1}^{\infty} R_{n}<\infty$ if and only if $\sum_{n=1}^{\infty} S_{n}<\infty$, where $S_{1}=1 / a_{2}$ and

$$
S_{n}=\frac{1}{a_{n+1}}+\frac{1}{a_{n+1}} \sum_{m=2}^{n}\left(d_{m}+\delta_{m n} b_{n}\right) S_{m-1}, \quad n \geq 2 .
$$

(Here, $\delta_{m n}$ is the Kronecker delta.) In addition, it is easily shown that $\left\{S_{n}\right\}$ satisfies (32) with $S_{0}=0$.

The final part follows because $Q^{*}$ is a birth-death $q$-matrix: (30) can have at most one linearly independent solution. Therefore, $\mathfrak{N}^{+}\left(\boldsymbol{Q}^{*}\right)=1$ when $\sum_{n=1}^{\infty} S_{n}<\infty$.

Remark 7. When $d_{n} \equiv 0$, Theorems 8 and 9 reduce to the well-known results for birth-death $q$-matrices, based on series commonly denoted $R$ and $S$; see, for example, Theorems 3.2.2 and 3.2.3 of [1].

Theorems 8 and 9 and Lemma 2 combine to give the following simple result.

Corollary 6. Let $\boldsymbol{Q}$ be the asymptotic birth-death q-matrix over $\mathbb{N}_{+}$whose restriction $\boldsymbol{Q}^{*}$ is determined by (31), and let $R$ and $S$ be the series defined in Theorems 8 and 9 , respectively. Then

(i) $Q$ is regular if and only if $R=\infty$, and

(ii) if $\boldsymbol{Q}$ is not regular then $\boldsymbol{Q}$ is quasi-regular if and only if $S<\infty$.

Our final example illustrates all of these results. It has the simplifying feature that both $d_{n} / a_{n}$ and $b_{n} / a_{n}$ do not depend on $n$.

Example 5. Suppose that $a_{n}=a n^{\theta}, b_{n}=b n^{\theta}$, and $d_{n}=d n^{\theta}, n \geq 1$, where $a>0, b>0$, $d>0$, and $\theta$ is any real number. After a modicum of algebra we find that

$$
R=\frac{1}{b}\left(\sum_{n=1}^{\infty} \frac{1}{n^{\theta}}+d \sum_{n=0}^{\infty}\left(\frac{a}{b}\right)^{n}\right) \sum_{n=0}^{\infty}\left(\frac{a}{b}\right)^{n} .
$$

So, by Corollary 6(i), $Q$ is regular if and only if $\theta \leq 1$ or $a \geq b$. 
In order to evaluate $S$ we set $T_{n}=a_{n+1} S_{n}$, meaning that the recursion (32) can be written more simply as

$$
T_{n+1}=\left(\frac{a_{n+1}+b_{n+1}+d_{n+1}}{a_{n+1}}\right) T_{n}-\frac{b_{n}}{a_{n}} T_{n-1}, \quad n \geq 1 .
$$

We then obtain $T_{n+1}=(1+b / a+d / a) T_{n}-(b / a) T_{n-1}, n \geq 1$, with $T_{0}=0$ and $T_{1}=a_{2} S_{1}=1$. The characteristic polynomial of this difference equation has the two real zeros

$$
\frac{a+b+d}{2 a} \pm \frac{\sqrt{(a-b)^{2}+d^{2}+2 a d+2 b d}}{2 a}
$$

(given by $3 / 2 \pm \sqrt{5} / 2$ if $a=b=d>0$ ). Denoting these zeros by $\lambda_{1}$ and $\lambda_{2}$, we can see that $0<\lambda_{1}<1<\lambda_{2}$. Thus, since $T_{0}=0$ and $T_{1}=1$, we obtain $T_{n}=\left(\lambda_{2}^{n}-\lambda_{1}^{n}\right) /\left(\lambda_{2}-\lambda_{1}\right)$, $n \geq 0$. We deduce that

$$
S_{n}=\frac{\lambda_{2}^{n}-\lambda_{1}^{n}}{a\left(\lambda_{2}-\lambda_{1}\right)(n+1)^{\theta}}, \quad n \geq 1,
$$

and, hence, that $S=\infty$. Corollary 6(ii) allows us to conclude that if $\boldsymbol{Q}$ is not regular (that is, $\theta>1$ and $a<b$ ), then $\boldsymbol{Q}$ is never quasi-regular.

\section{Acknowledgements}

We would like to thank the referee for valuable comments and suggestions, which lead to a much improved presentation of our results. The support of the Australian Research Council (grant no. A00104575) is gratefully acknowledged. The work of Ben Cairns is supported by a $\mathrm{PhD}$ scholarship from the Australian Research Council Centre of Excellence for Mathematics and Statistics of Complex Systems.

\section{References}

[1] Anderson, W. J. (1991). Continuous-Time Markov Chains: An Applications-Oriented Approach. Springer, New York.

[2] Brockwell, P. J. (1985). The extinction time of a birth, death and catastrophe process and of a related diffusion model. Adv. Appl. Prob. 17, 42-52.

[3] Brockwell, P. J. (1986). The extinction time of a general birth and death process with catastrophes. J. Appl. Prob. 23, 851-858.

[4] Brockwell, P. J., Gani, J. And Resnick, S. I. (1982). Birth, immigration and catastrophe processes. Adv. Appl. Prob. 14, 709-731.

[5] Chen, A. Y. (2002). Uniqueness and extinction properties of generalized Markov branching processes. J. Math. Anal. Appl. 274, 482-494.

[6] Chen, A. Y. and Renshaw, E. (1990). Markov branching processes with instantaneous immigration. Prob. Theory Relat. Fields 87, 204-240.

[7] Chen, A. Y. and Renshaw, E. (1993). Existence and uniqueness criteria for conservative uni-instantaneous denumerable Markov processes. Prob. Theory Relat. Fields 94, 427-456.

[8] Chen, M. F. (1992). From Markov Chains to Nonequilibrium Particle Systems. World Scientific, Singapore.

[9] Chen, M. F. (1999). Single birth processes. Chinese Ann. Math. Ser. A 20, 77-82.

[10] Chen, M. F. and Zheng, X. G. (1983). Uniqueness criterion for $q$-processes. Sci. Sinica Ser. A 26, 11-24.

[11] Chen, R. R. (1997). An extended class of time-continuous branching processes. J. Appl. Prob. 34, 14-23.

[12] FelleR, W. (1940). On the integro-differential equations of purely discontinuous Markoff processes. Trans. Amer. Math. Soc. 48, 488-515.

[13] Hart, A. G. and Pollett, P. K. (1996). Direct analytical methods for determining quasistationary distributions for continuous-time Markov chains. In Athens Conf. on Applied Probability and Time Series Analysis, Vol. 1 (Lecture Notes Statist. 114), eds C. C. Heyde et al., Springer, New York, pp. 116-126. 
[14] Hart, A. G. and Pollett, P. K. (2000). New methods for determining quasi-stationary distributions for Markov chains. Math. Comput. Modelling 31, 143-150.

[15] Hou, C. T. (1974). The criterion for uniqueness of a $Q$-process. Sci. Sinica 17, 141-159.

[16] Hou, Z. T. and Guo, Q. F. (1988). Homogeneous Denumerable Markov Processes. Springer, Berlin.

[17] Pakes, A. G. (1986). The Markov branching-catastrophe process. Stoch. Process. Appl. 23, 1-33.

[18] Pollett, P. K. (1991). Invariant measures for $Q$-processes when $Q$ is not regular. Adv. Appl. Prob. 23, $277-292$.

[19] Pollett, P. K. (2001). Quasi-stationarity in populations that are subject to large-scale mortality or emigration. Environ. Internat. 27, 231-236.

[20] Pollett, P. K. and Taylor, P. G. (1993). On the problem of establishing the existence of stationary distributions for continuous-time Markov chains. Prob. Eng. Inf. Sci. 7, 529-543.

[21] Reuter, G. E. H. (1957). Denumerable Markov processes and the associated contraction semigroups on $l$. Acta Math. 97, 1-46.

[22] Reuter, G. E. H. (1976). Denumerable Markov processes. IV. On C. T. Hou's uniqueness theorem for $Q$-semigroups. Z. Wahrscheinlichkeitsth. 33, 309-315.

[23] Yan, S. J. And Chen, M. F. (1986). Multidimensional Q-processes. Chinese Ann. Math. Ser. A 7, 90-110.

[24] Zhang, J. K. (1984). Generalized birth-death processes. Acta Math. Sinica 46, 241-259 (in Chinese).

[25] Zhang, Y. H. (2001). Strong ergodicity for single-birth processes. J. Appl. Prob. 38, $270-277$. 